\title{
Temperature sensor using fiber ring laser based on a core-offset Mach-Zehnder interferometer
}

\author{
M. A. Contreras-Terán*, J. M. Sierra-Hernández, D. Jauregui-Vázquez, and R. Rojas-Laguna \\ División de Ingenierías Campus Irapuato Salamanca, \\ Departamento de Electrónica, Universidad de Guanajuato, Salamanca 36885, México. \\ *e-mail: ma.contrerasteran@ugto.mx
}

Received 11 December 2020; accepted 13 February 2021

\begin{abstract}
The development of fiber optic sensors receives particular interest since they can be used in multiple applications. As a result, fiber optic laser-sensor schemes had emerged as reliable devices due to their characteristics such as high power, low threshold, and high stability. In this work, a fiber laser-sensor based on a ring cavity configuration is experimentally proposed. The fiber laser cavity is operated by a core-offset Mach-Zehnder interferometer used as a wave selection filter and temperature detection device. Here, the proposed fiber laser-sensor exhibits sensing properties such as the sensitivity of $0.04933 \mathrm{~nm} /{ }^{\circ} \mathrm{C}$, with a dynamic range of $90^{\circ} \mathrm{C}$ and a signal-to noise ratio of $53 \mathrm{~dB}$.
\end{abstract}

Keywords: Temperature sensor; fiber óptics, láser, Mach-Zehnder.

\section{Introduction}

One of the most significant advances in lasers is related to the improved techniques in manufacturing fiber optics; here, new pumping sources and the use of rare earth improve the fiber optic laser's performance [1]. The fiber optic laser presents some interesting characteristics such as tunable and multiple wavelengths emissions; thus, several schemes have been proposed. The main component to obtaining tunable and multiple wavelengths laser emission is the wavelength selector filter (SWF, Selective wavelength Filter). This filter is implemented in different ways, such as using Fiber Bragg Gratings(FBG) [2,3], Fabry-Perot interferometers (FPI) [4,5], Sagnac interferometers [6,7], and Mach-Zehnder interferometer(MZI) [8-11].

Within the literature, different types of multi-wavelength lasers are presented using MZI [9]; in this case, a ring configuration is reported, the fiber optic laser uses Ytterbium Doped Fiber (YDF) as a gain medium. This fiber laser was able to emit one, two, three, and four peaks, and its emission range was from 1030 to $1037 \mathrm{~nm}$. The MZI proposed in this work was implemented by cascading two 50/50 couplers to form the interferometer's arms. The technique to control the multiple emissions was by adjusting the polarization state (PC) into the cavity; a Polarization Controller adjusts this parameter.

It is essential to notice that for almost 20 years, fiber optic laser had been applied in various fields such as; spectroscopy, optical processing, multiplexing of communication systems and sensors [12]. The sensing application is attractive, mainly because it has a high signal-to-noise sensitivity [13]. However, these lasers are not used as sensors since filters are sensitive to unstable modal competition caused by the homogeneous widening at the output spectrum of erbiumdoped fiber [14]. It is important to mention that to obtain a linear response, the laser emissions require other optical components. This work proposes a wave selection filter based on a Mach-Zehnder interferometer; this filter converts temperature changes into laser emission changes in a linear range.

\section{Methodology}

A flow chart is presented in Fig. 1 to describe the fiber opticlaser sensor procedure.

\subsection{Erbium-doped fiber characterization}

Q-Photonics semiconductor was used at the maximal current of $350 \mathrm{~mA}$ to pumping an Erbium-doped fiber. The experi-

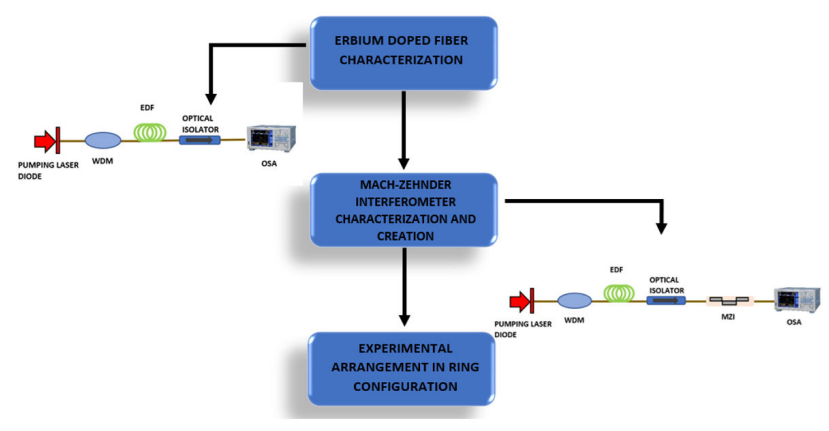

FIGURE 1. Flow diagram of the implemented schemes.

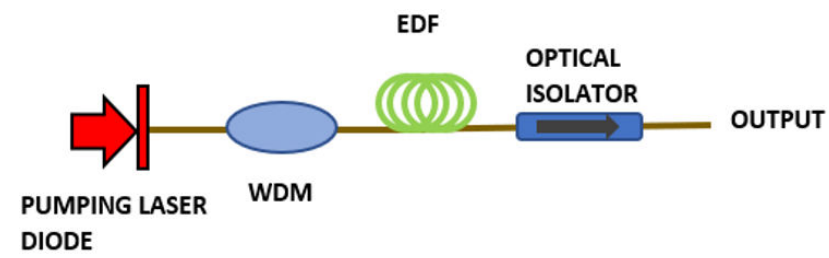

FIGURE 2. Erbium-doped fiber amplifier scheme. 


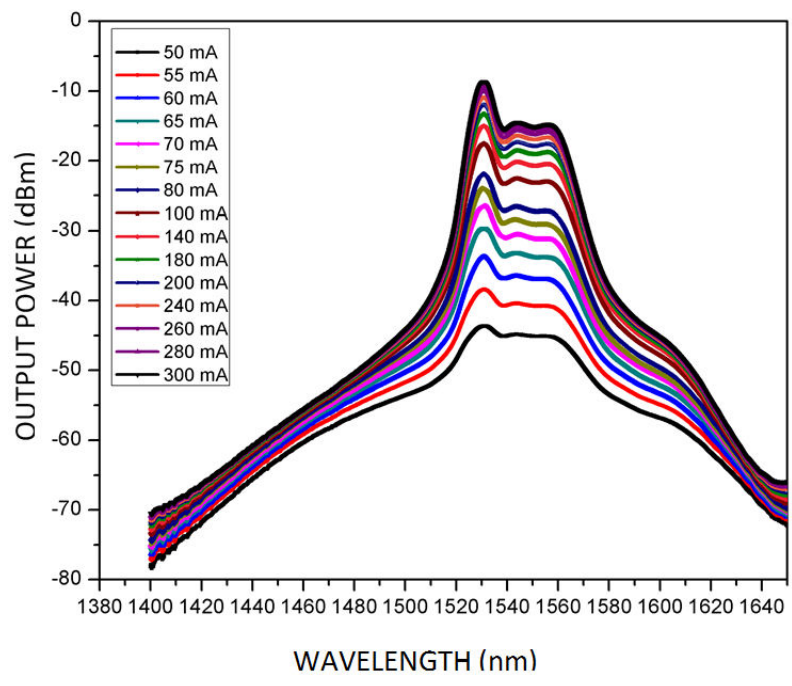

FIGURE 3. Spectral erbium-doped fiber response for different pumping currents.

mental setup used to analyze the doped fiber is presented in Fig. 2.

The function of WDM (wavelength division multiplexing) is to allow the control of the wavelength, and as a result, it is possible to observe the performance of the Erbium-doped fiber; here, the pump power was increased every $10 \mathrm{~mA}$ until reaching $300 \mathrm{~mA}$. The Erbium-doped fiber spectrum is presented in Fig. 3. As can be observed, the spectrum provides a uniform and flat spectrum in the range of $1540 \mathrm{~nm}$ to $1570 \mathrm{~nm}$; thus, it can be inferred that this region will be optimal to observe a laser emission due to the minimal modal competition will be presented. However, it is necessary to analyze the filter response to guarantee minimal modal competition.

\subsection{Fiber Optic Mach-Zehnder Optical Filter}

The schematic configuration of the M-Z core offset structure is presented in Fig. 4. Some parameters govern this structure; here, the total length $(L)$ plays an important role. The operation is as follows; the pumping light is divided at the moment to arrive at the first core-offset section, here the pumping light travels in two regions: core and cladding, at the end of the last section, the light is coupled to the core, at this point an interference is generated.

The joints are made employing an arc electric fusion joint. The offset implemented was $30 \mu \mathrm{m}$ in each of the arms. The creation stage can be better appreciated in Fig. 5, where

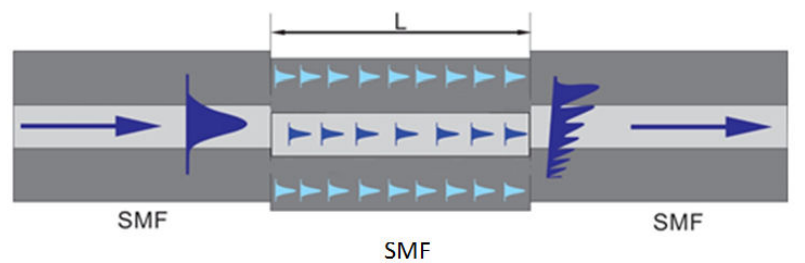

FIGURE 4. Mach-Zehnder interferometer scheme.

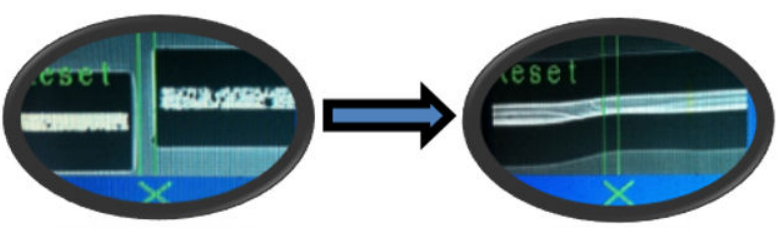

FIGURE 5. Splicer view at the moment to generate the optical fiber interferometer.

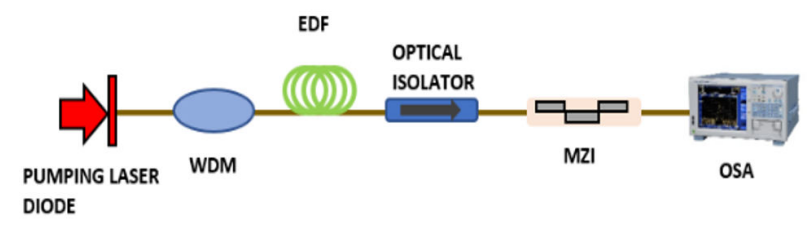

FIGURE 6. Scheme for testing Mach-Zehnder interferometers.

the applied phase shift and the joint inside the electric arc splicer can be observed.

The scheme used to test the interferometers is shown in Fig. 6.

The scheme presented in Fig. 6 was used to characterize all the fabricated interferometers were characterized; their response is presented in Fig. 7. The fabrication process reveals that the optimal offset of $30 \mu \mathrm{m}$ provides a good interference spectrum with $\mathrm{a} \sim 10 \mathrm{~dB}$ power output. As can be appreciated, both interferometers have different $\Delta \lambda$ and visibility; as a result, it can be expected different responses due to the modal competition will be altered. The interferometer with a $6 \mathrm{~cm}$ length has a visibility of $10 \mathrm{~dB}$ and $\Delta \lambda \sim 6 \mathrm{~nm}$; in contrast, the interferometer with a small length has visibility of $5.5 \mathrm{~dB}$ and $\Delta \lambda \sim 7 \mathrm{~nm}$.

The following relation governs the proposed optical fiber interferometer

$$
I=I 1+I 2+2 I 1 I 2 \cos 2 \pi([\mathrm{nco}-\mathrm{ncl}] / \lambda L),
$$

where $I 1$ and $I 2$ are the core and cladding modes generated at the core-offset region. This expression generates an oscillating signal where the phase depends on the effective refractive index difference of the modes involve (nco $-\mathrm{ncl})$, here, nco represents the effective refractive index of the core and $n c l$ of the cladding; moreover, the cavity length $(L)$ and the wavelength operation $(\lambda)$ are also considered. An interference point $(\lambda D)$ depends on the following relation:

$$
\lambda D=2(\mathrm{nco}-\mathrm{ncl}) L /(2 k+1),
$$

here, $K$ is an arbitrary integer that is related to the number of modes. As can be appreciated, the interferometer's length plays an important role in the interference pattern, and this parameter will affect the free spectral range.

\subsection{Temperature response of Mach-Zehnder interfer- ometer}

Before the final configuration, the thermal response of the MZI is analyzed. Here, the temperature was altered by the 

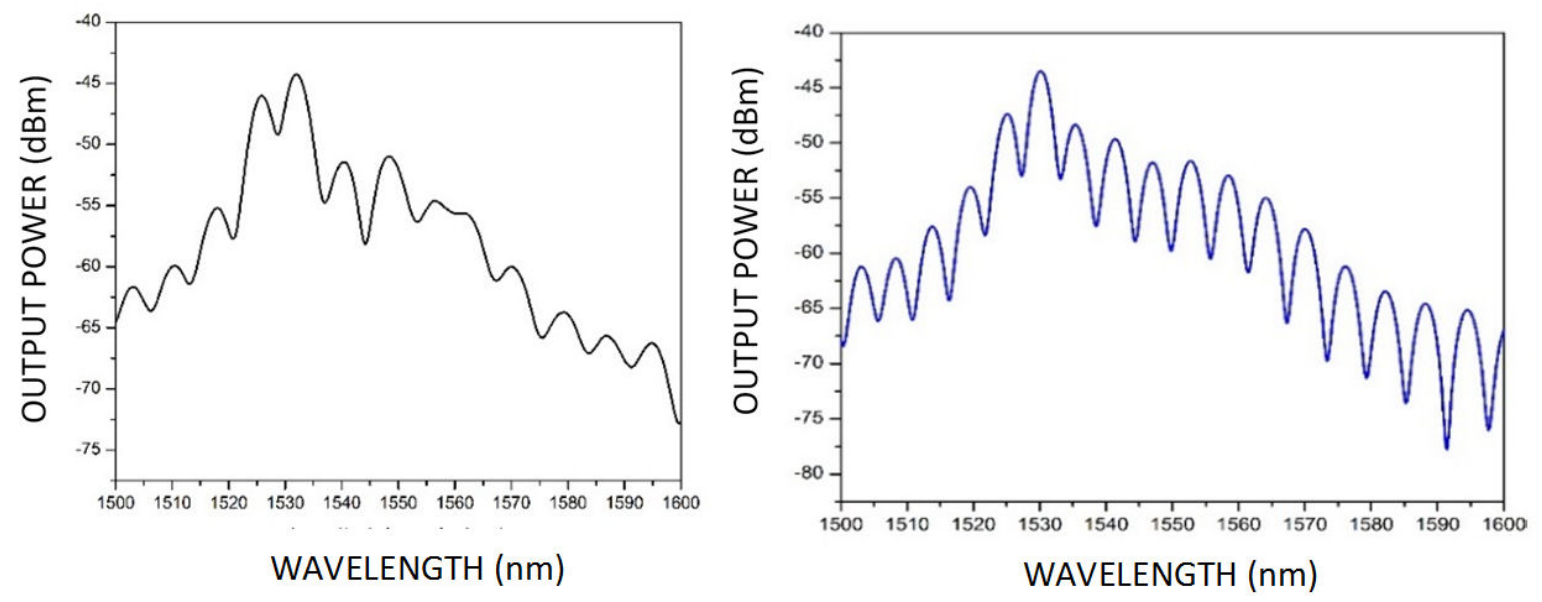

FIgURE 7. a) Spectrum of M-Z with a $4.5 \mathrm{~cm}$ length, b) a $6 \mathrm{~cm}$ length $M-Z$ spectrum.

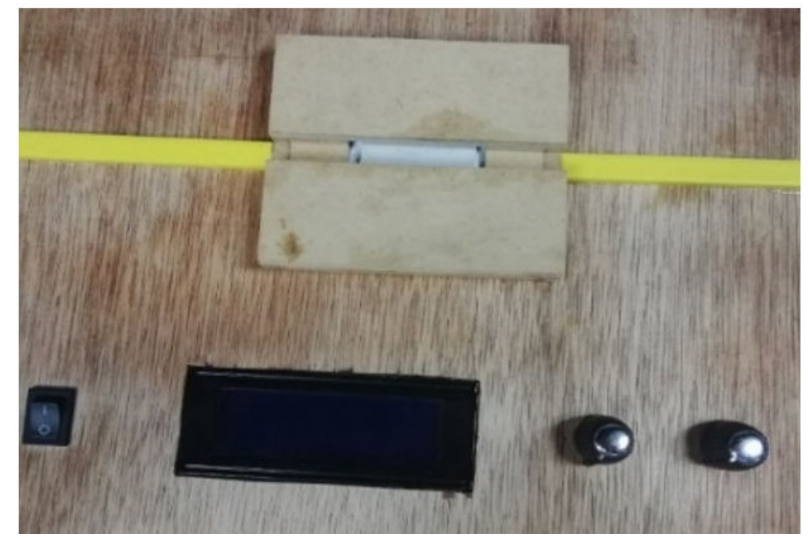

FIGURE 8. Temperature controller used for thermal analysis.

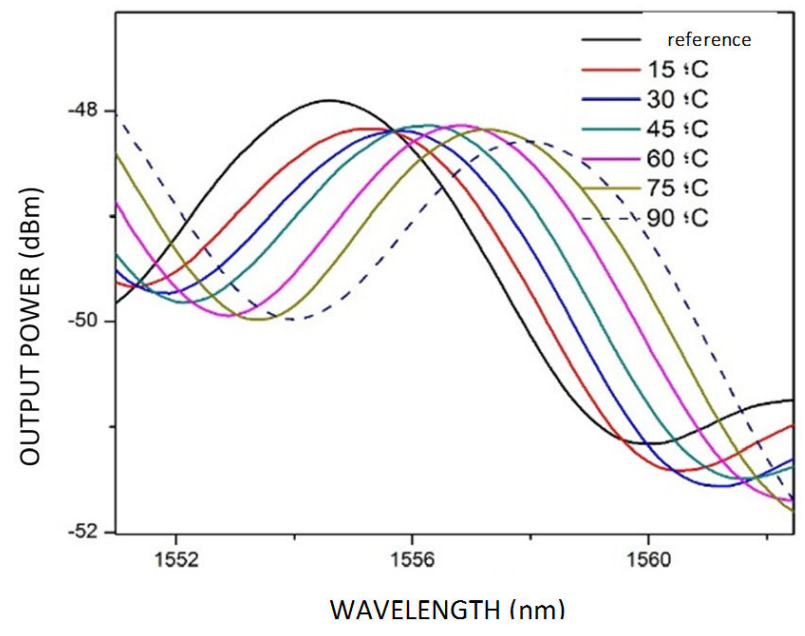

FIGURE 9. Temperature responses of MZI with a length of $6 \mathrm{~cm}$.

temperature controller (see Fig. 8). As a result, the MZI exhibits a precise wavelength shifting to longer wavelengths. (see Fig. 9). The interference peak centered at $1554.5 \mathrm{~nm}$ is shited to $1558.3 \mathrm{~nm}$; thus, a total wavelength shifting of $3.8 \mathrm{~nm}$ is presented for a temperature variation of $90^{\circ} \mathrm{C}$, as a consequence, a sensitivity of $0.04222 \mathrm{~nm} /{ }^{\circ} \mathrm{C}$ can be expected. The interference fringes have minimal power variations. Its response is not linear; however, these aspects alter the modal competition into a ring fiber laser cavity; thus, it can be expected a multi-wavelength emission and tunable effect.

\subsection{Laser scheme in a ring configuration}

At this point, the WSF was analyzed to be implemented into the ring laser cavity; the phase modulation presented in Fig. 11 can be used to modulate the laser response. The ring fiber-optic laser configuration is shown in Fig. 10. In this figure, we can observe the components used to achieve laser emission. The laser is composed of the semiconductor QPHOTONICS, which has a central wavelength at $980 \mathrm{~nm}$, followed by a WDM junction; here, the port $1550 \mathrm{~nm}$ is used. An erbium-doped fiber of approximately a total length of $4 \mathrm{~m}$ is used as an active medium, here, the erbium-doped fiber generates a spectrum in the range of 1500 to $1600 \mathrm{~nm}$, in turn to a 90/10 coupler to guarantee a coupling union of signals and allow to monitor the signal, at the same time the signal continue transmitting the 90 percent in the closed system. Moreover, a Mach Zehnder core offset interferometer is connected as a WSF; also, an optical isolator is spliced to

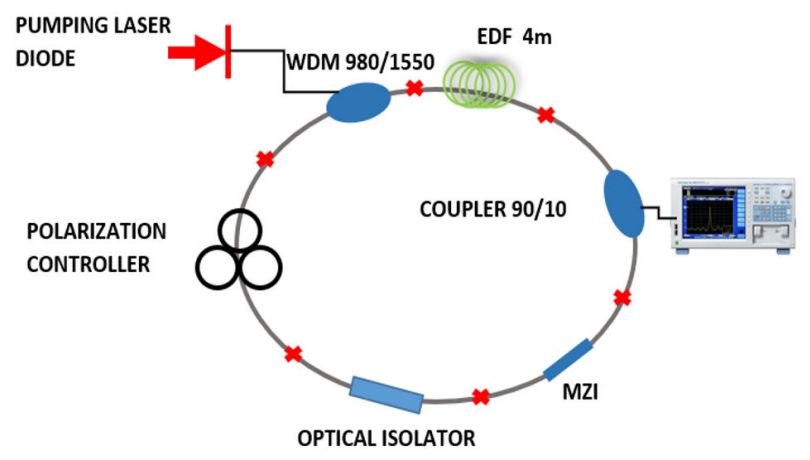

FIGURE 10. Experimental ring laser arrangement configuration. 


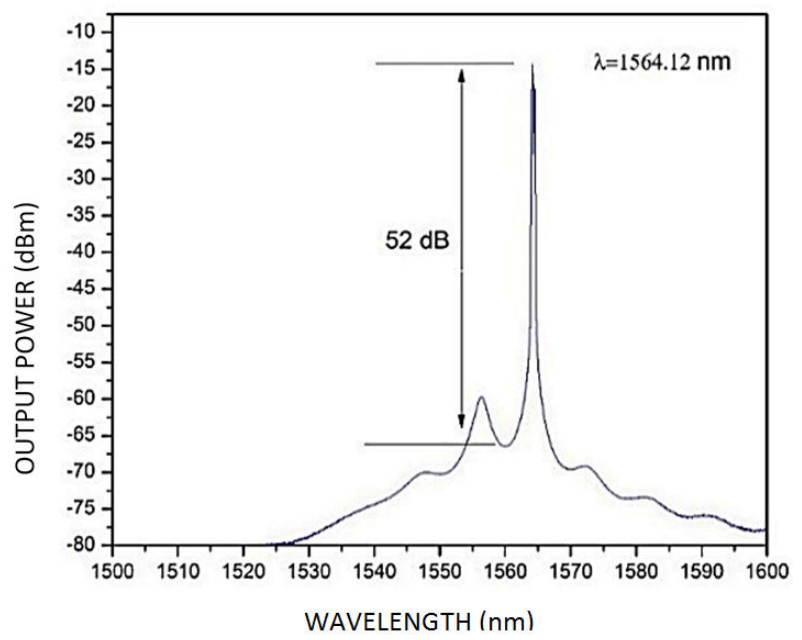

FIGURE 11. Emission laser result of $4.5 \mathrm{~cm}$ MZI.

ensure one direction of the light, followed by a polarization controller used to reduce instabilities and closing the ring cavity. The laser response is observed at the output coupler employing the YOKOGAWA AQ630B optical spectrum analyzer.

\section{Results}

\subsection{Fiber-optic laser results}

By using an optical fiber MZI, with a length of $4.5 \mathrm{~cm}$, into the configuration presented in Fig. 10, a single line emission is achieved, the emission is centered at $1564 \mathrm{~nm}$ and presents a Side Mode Suppression Ratio (SMSR) of $52 \mathrm{~dB}$, this initial spectrum can be observed in Fig. 11.

By modifying the interferometer's length, the spectrum also suffers an emission change; Fig. 12 shows the spectrum generated by a $6 \mathrm{~cm}$ interferometer. Here, dual laser emission is achieved, and the maximal output power presented is $53 \mathrm{~dB}$.

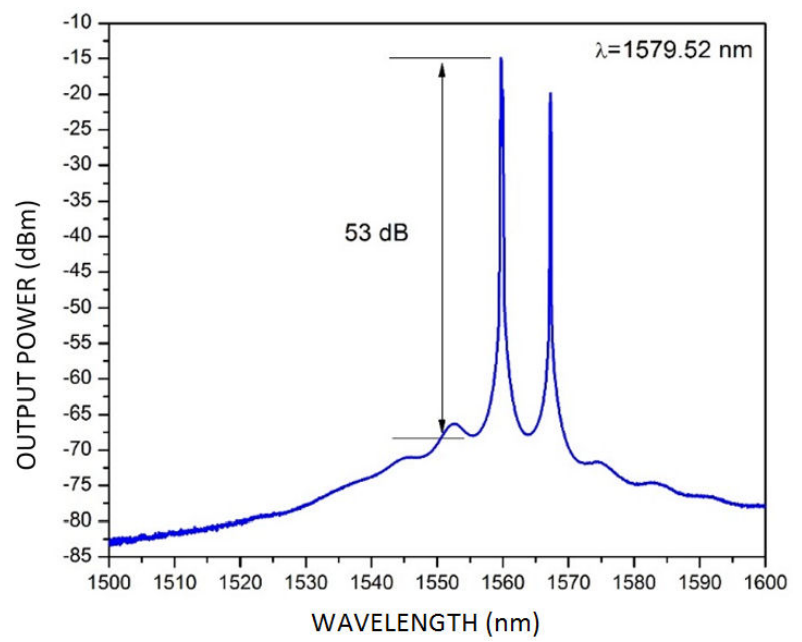

FIGURE 12. Simultaneous emission of two laser lines.

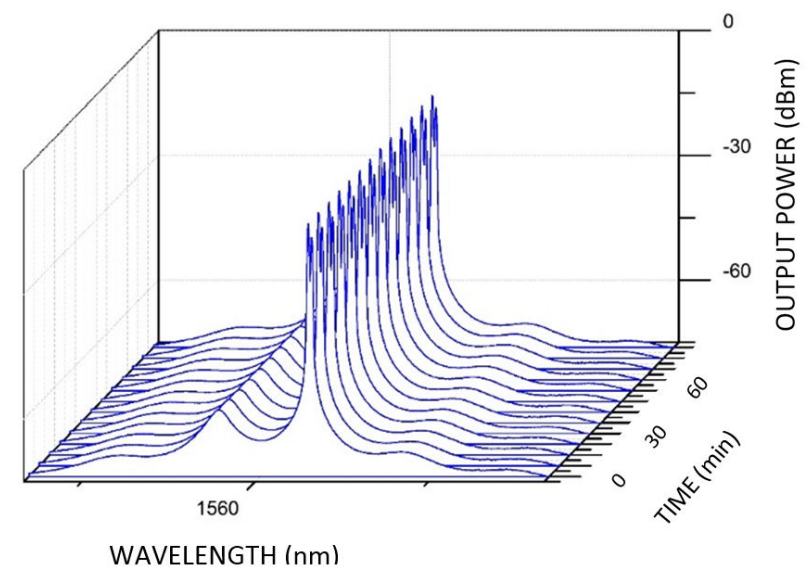

FIGURE 13. Fiber optic laser stability results using $4.5 \mathrm{~cm}$ interferometer length.

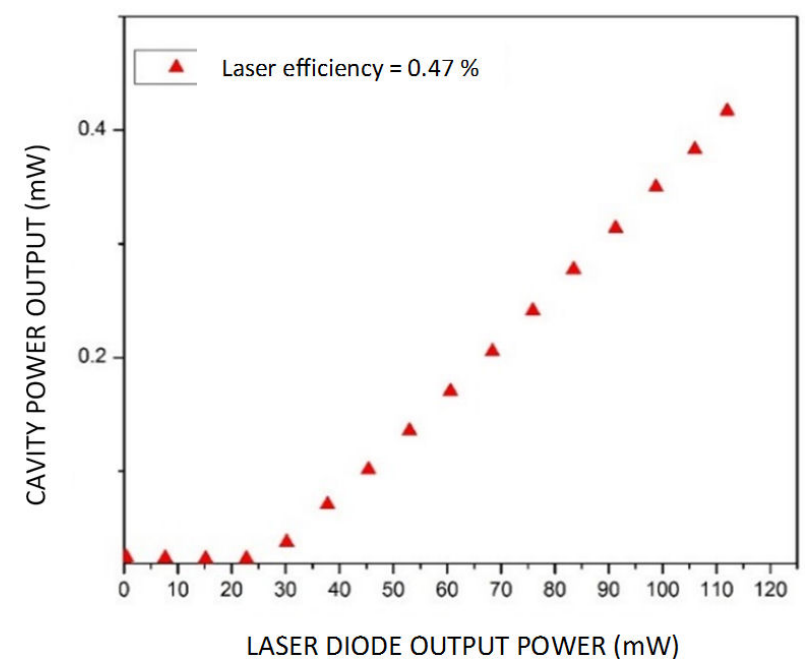

FIGURE 14. Fiber-optic laser efficiency of $4.5 \mathrm{~cm}$ interferometer length.

\subsection{Laser stability and efficiency results}

Two parameters are essential to describe the performance of the fiber laser: stability and efficiency. Consequently, the spectrum of the $4.5 \mathrm{~cm}$ interferometer length is recorded every 5 minutes during an hour, and the response is presented in Fig. 13. This stability analysis shows minimal power and wavelength fluctuations. Moreover, the efficiency was computed and presented in Fig. 14. Here, a laser efficiency of 47 percent and a laser power of $0.435 \mathrm{~mW}$ can be observed.

In Fig. 15, we can observe a double laser emission for a 6 $\mathrm{cm}$ MZI which was monitored for $60 \mathrm{~min}$, obtaining changes in the output power around $\sim 0.4 \mathrm{~dB}$ approximately and obtaining a laser efficiency of 46 percent, with a power of 0.42 $\mathrm{mW}$, as shown in Fig. 16.

\subsection{Tunable response for temperature variation}

Due to the good performance of the cavity and the filter, a thermal sensor application is explored. Hence, temperature 


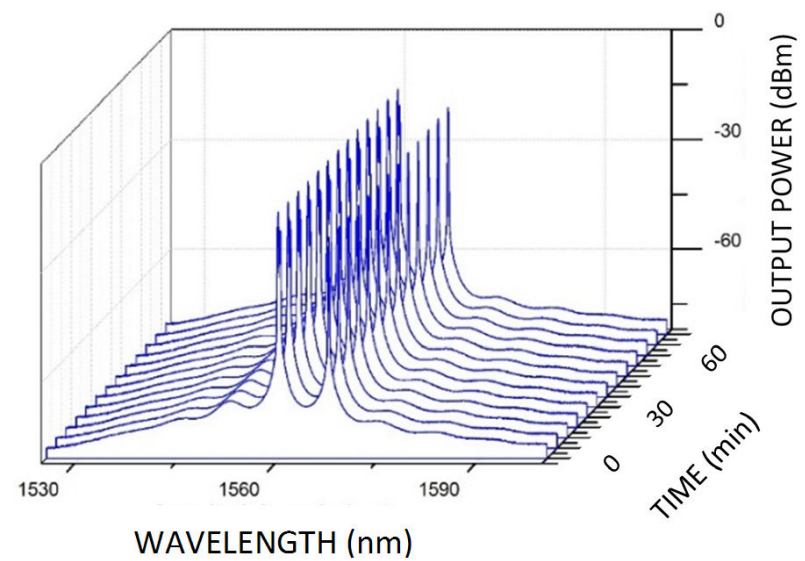

FIGURE 15. Result of fiber optic laser stability using $6 \mathrm{~cm}$ interferometer and dual emission.

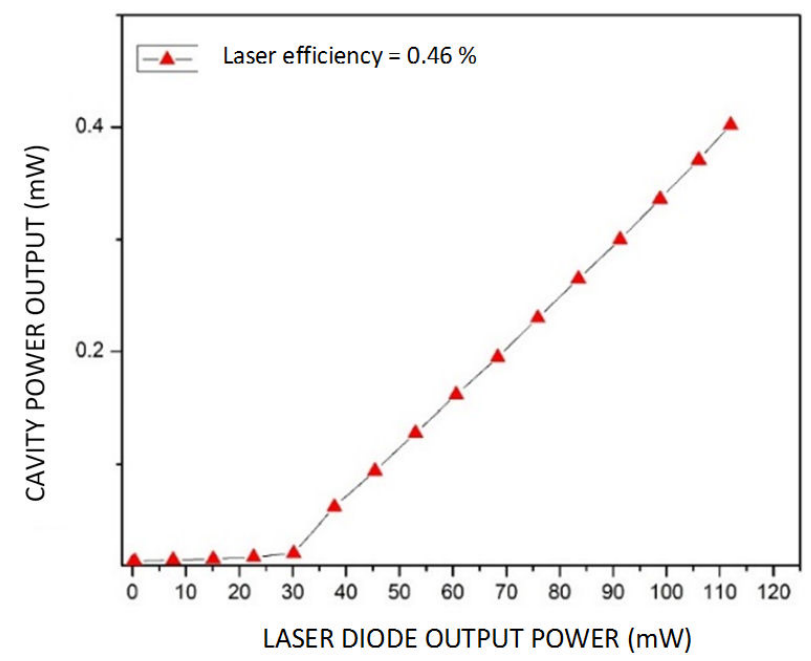

FIGURE 16. Fiber-optic laser efficiency with $6 \mathrm{~cm}$ interferometer.

variations were applied by using the arrangement and procedure described above (Sec. 2.3). As a result, an apparent wavelength shifting is presented.

The output laser spectrum was recorded every 5 degrees for visual purposes. Figure 17 shows the variations every 15 degrees, with a linear shift of just under $1 \mathrm{~nm}$ of shift every 15 degrees. In the same way, the results are reported in Fig. 18 for the $6 \mathrm{~cm}$ interferometer. As can be appreciated, the single lasing line presents a wavelength shifting to a longer wavelength; here, the lasing mode presents a constant SMSR around $45 \mathrm{~dB}$.

The analyses show a peculiarity in the laser emission; here, the single lasing line is tuned $0.8 \mathrm{~nm}$ when the temperature is increased by $15^{\circ} \mathrm{C}$ steps, resulting in sensitivity around $0.04933 \mathrm{~nm} /{ }^{\circ} \mathrm{C}$ is achieved. A particular characteristic to be considered for multiwavelength laser emissions is related to the interferometer's length. As the silica area increases, the modal competition is more susceptible to temperature variations. This effect can be appreciated in Fig. 18.

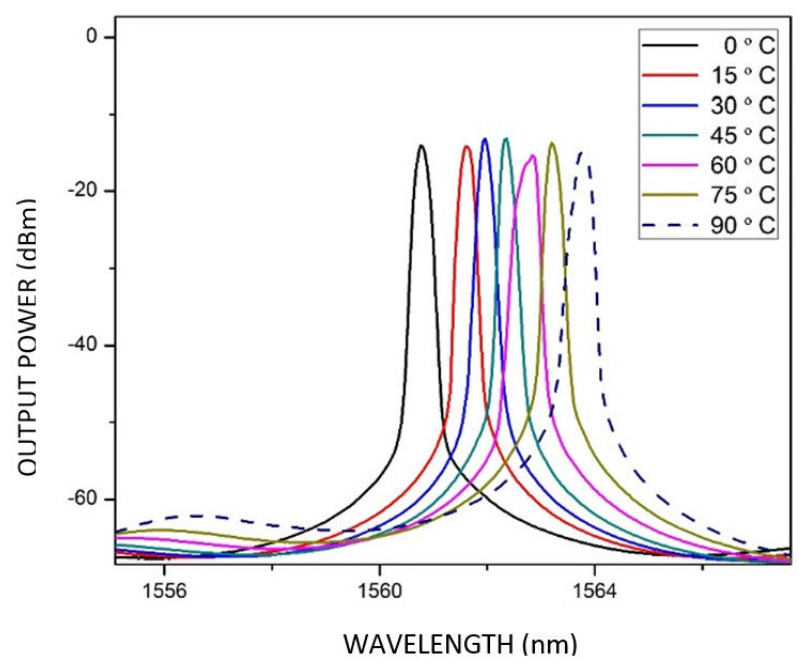

FIGURE 17. Fiber laser tuning effect between 1560-1564 nm.

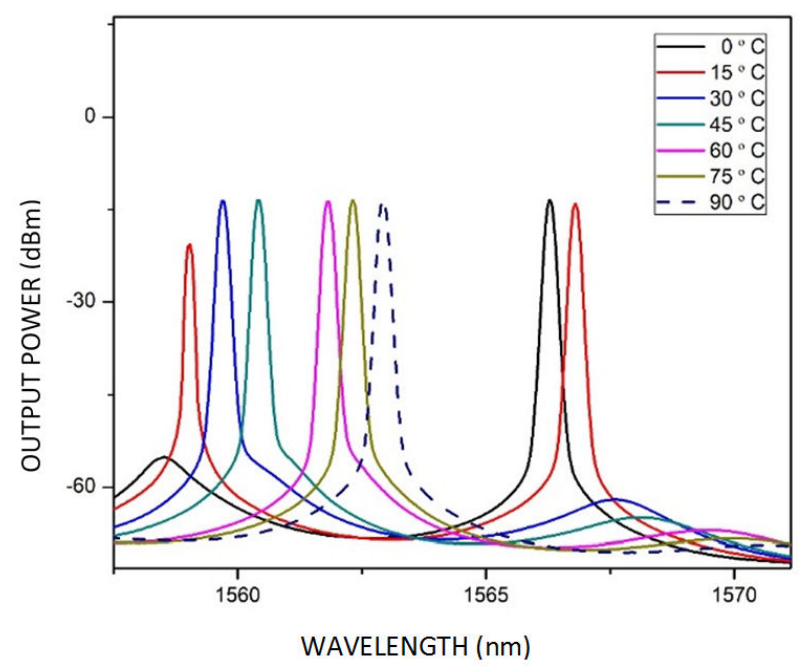

FIGURE 18. Output laser tuning effect between 1558-1563 nm.

A dual-wavelength emission starts from minimal temperature variation $\left(15^{\circ} \mathrm{C}\right)$. This thermal change generates two lasing modes between 1559 and $1567 \mathrm{~nm}$.

\subsection{Linearity in the laser cavity}

A study was carried out regarding the linearity on the temperature variation to observe the degree of linear adjustment; the wavelength shifting is presented in Fig. 19; as can be observed, the laser emission peak within exhibits a linear response as the temperature is increased.

The sensitivity of the fiber laser-based o different length MZI is $0.03333 \mathrm{~nm} /{ }^{\circ} \mathrm{C}$ and $0.04933 \mathrm{~nm} /{ }^{\circ} \mathrm{C}$.

Tables I and II present an excellent summary of both the laser emission peak in each of the interferometers when applying the temperature phenomenon and the degree of linear adjustment, presented in Fig. 19. 

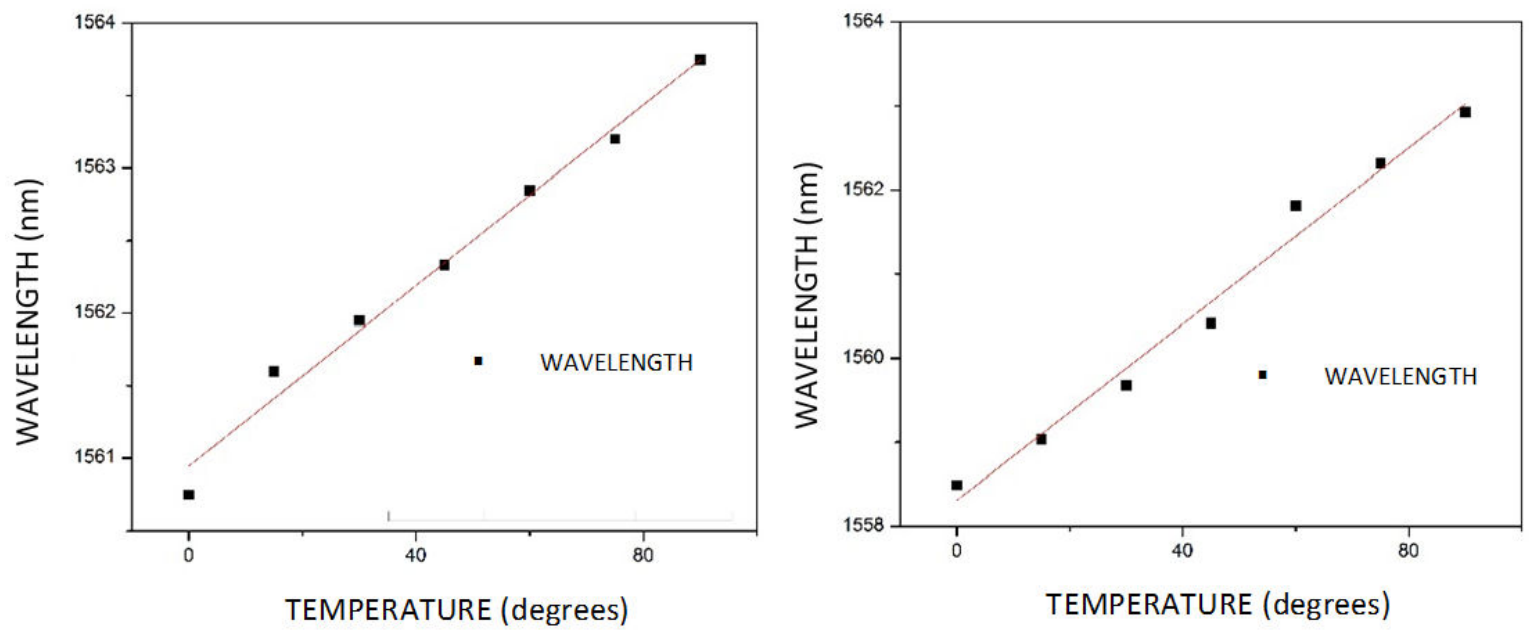

FIGURE 19. Comparative MZ interferometer shifting response a) $4.5 \mathrm{~cm}$ and b) $6 \mathrm{~cm}$ for thermal variation.

TABLE I. Fiber laser linear adjustment results.

\begin{tabular}{|c|c|c|}
\hline \multicolumn{2}{|c|}{ Linear fit } & Standard deviation \\
\hline Laser $6 \mathrm{~cm}$ : & 0.98009 & 0.16472 \\
\hline Laser $4.5 \mathrm{~cm}$ : & 0.98332 & 0.08958 \\
\hline
\end{tabular}

TABLE II. Wavelength temperature changes for both analyzed interferometers.

\begin{tabular}{cccccccc}
\hline Interferometer $(\mathrm{cm})$ & \multicolumn{7}{c}{ Wavelength variation $(\mathrm{nm})$} \\
\hline 4.5 & Reference- & $15^{\circ}-$ & $30^{\circ}-$ & $45^{\circ}-$ & $60^{\circ}-$ & $75^{\circ}-$ & $90^{\circ}-$ \\
& 1560.75 & 1561.60 & 1561.95 & 1562.33 & 1562.85 & 1563.2 & 1563.75 \\
\multirow{2}{*}{6} & Reference- & $15^{\circ}-$ & $30^{\circ}$ & $45^{\circ}-$ & $60^{\circ}-$ & $75^{\circ}-$ & $90^{\circ}-$ \\
& 1558.49 & 1559.03 & 1559.68 & 1560.41 & 1561.82 & 1562.31 & 1562.93 \\
\hline
\end{tabular}

\section{Conclusions}

Finally, In this work, a ring fiber optic laser-based on coreoffset MZI was experimentally demonstrated. The development of single-mode fiber optic sensors is presented here, the creation of an optical filters Mach-Zehnder structure is presented under the core offset technique with a $30 \mu \mathrm{m}$ gap between body and arms, made in manual manufacturing, the MZI used as a wave selecting a filter and validated as a temperature tunable device. This allowed us to develop a laser in a ring configuration entirely of fiber optic, presenting the response in stability and efficiency, obtaining average laser efficiencies of 40 percent, and stable average powers of
$50 \mathrm{~dB}$, and a linear fit over 0.98 in temperature tune. Furthermore, a laser temperature sensor based on a core-offset Mach-Zehnder interferometer is presented. The experimental results showed a temperature sensitivity of $0.04933 \mathrm{~nm} /{ }^{\circ} \mathrm{C}$ with a dynamic range of $90^{\circ} \mathrm{C}$ and a signal-to-noise ratio of $53 \mathrm{~dB}$.

\section{Acknowledgments}

This work was partially supported by the Universidad de Guanajuato and directly supported by the Consejo Nacional de Ciencia y Tecnología (CONACyT) for the grant: Marco Antonio Contreras Teran-966683.
1. H. Simon, and W. Colin, Laser Physics, (Oxford University press 2010).

2. C. H. Yeh and C. W. Chow, Single-longitudinal-mode erbiumdoped fiber laser with novel scheme utilizing fiber Bragg grating inside ring cavity, Laser Physics 20 (2010) 512. https:

\section{//doi.org/10.1134/S1054660X10030229}

3. A. Hamzah et al., Compact fiber laser at L Band region using erbium-doped zirconia fiber, Laser Physics 21 (2011) 176. https://doi.org/10.1134/S1054660X10230040 
4. S. Pan, C. Lou and Y. Gao, Multiwavelength erbium-doped fiber laser on inhomogeneous loss mechanism by use highly nonlinear fiber and a Fabry-Perot filter, Optics Express 14 (2006) 1113. https://doi.org/10.1364/OE.14. 001113

5. F. F. Zhong, Y. Xu, Y. J. Zhang and Y. Ju, Widely ultra-narrow linewidth $104 \mathrm{~nm}$ tunable all-fiber compact erbium-doped ring laser, Laser Physics 21 (2011) 219. https://doi.org/ $10.1134 / \mathrm{S} 1054660 \times 11010269$

6. R. K. Kim and Y. G. Han, Switchable multiple lasing oscillations in an erbium-doped fiber ring laser using a single stage of a Sagnac loop mirror, Applied Physics B 103 (2011) 813. https://doi.org/10.1007/s00340-011-4529-9

7. G. Sun, Y. Hu, Y. Zhou and Y. Chung, Broad tunability of erbium-doper fiber ring laser based on few-mode polarization maintaining fiber Sagnac interferometer, Applied Physics B 102 (2011) 589. https : //doi.org/10.1016/ j.optlastec.2009.08.008

8. A. Castillo-Guzman et al., Widely tunable-erbium doped fiber laser based on multimode interference effect, Optics Express 18 (2010) 591. https://doi.org/10.1364/OE.18. 000591

9. Ch. Tu, W. Guo, Y. Li, S. Zhang and F. Lu, Stable multiwavelength and passively mode-locked Yb-doped fiber based on nonlinear polarization rotation, Optics Communications $\mathbf{2 8 0}$ (2007) 448.
10. J. Zhang et al., A tunable erium-doped fiber laser based on an MZ interferometer and a birefringence fiber filter, Journal of Photonics A 14 (2012) 015402. https: / / doi .org/10. $1088 / 2040-8978 / 14 / 1 / 015402$

11. W. G. Chen et al., Switchable multi-wavelength fiber ring laser based on a compact in-fiber Mach-Zehnder interferometer with photonic crystal fiber, Laser Physics 19 (2009) 2115. https : //doi.org/10.1134/S1054660X09210026

12. M.N. Mohd Nasir, M.H. Al-Mansoori, H.A. Abdul Rashid, P.K. Choudhury, and Z. Yusoff, Multiwavelength Brillouinerbium fiber laser incorporating a fiber Bragg grating filter, Laser Phys. 18 (2008) 446. https://doi.org/10. 1134/s11490-008-4016-0

13. J.M. Sierra-Hernandez et al., A tunable multi-wavelength erbium-doped fiber laser based on a Mach-Zehnder interferometer and photonic crystal fiber, Laser Phys. 23 (2013) 125103. https://doi.org/10.1088/1054-660X/23/12/ 125103

14. M. Bianchetti et al., Switchable multi-wavelength laser based on a core-offset Mach-Zehnder interferometer with non-zero dispersion-shifted fiber, Opt. and Laser Technol. 104 (2018) 49. https://doi.org/10.1016/j. optlastec.2018.02.012 